\title{
The thermospheric effects of a rapid polar cap expansion
}

\author{
D. W. Idenden \\ School of Mathematics and Statistics, The Hicks Building, University of Sheffield, Sheffield, S3 7RH, UK
}

Received: 27 October 1997 / Revised: 4 June 1998 / Accepted: 6 June 1998

\begin{abstract}
In a previous publication we used results from a coupled thermosphere-ionosphere-plasmasphere model to illustrate a new mechanism for the formation of a large-scale patch of ionisation arising from a rapid polar cap expansion. Here we describe the thermospheric response to that polar cap expansion, and to the ionospheric structure produced. The response is dominated by the energy and momentum input at the dayside throat during the expansion phase itself. These inputs give rise to a large-scale travelling atmospheric disturbance (TAD) that propagates both antisunward across the polar cap and equatorward at speeds much greater than both the ion drifts and the neutral winds. We concentrate only on the initially poleward travelling disturbance. The disturbance is manifested in the neutral temperature and wind fields, the height of the pressure level surfaces and in the neutral density at fixed heights. The thermospheric effects caused by the ionospheric structure produced during the expansion are hard to discern due to the dominating effects of the TAD.
\end{abstract}

Key words. Ionosphere (ionosphere-atmosphere interaction; modeling and forecasting; plasma convection).

\section{Introduction}

The Sheffield/UCL/SEL coupled thermosphere-ionosphere-plasmasphere (CTIP) model (Fuller-Rowell et al., 1996; Millward et al., 1996) employing a new time-dependent high-latitude electric field model (Idenden et al., 1996) has been used to examine the ionospheric effects of a rapid polar cap expansion during winter solstice (Idenden et al., 1997). Rapid ion flows through the dayside convection throat cause a depletion at the F2 peak in the tongue of ionisation (TOI). The
TOI is formed at certain UTs when the proximity of the polar cap to the solar terminator allows solar produced plasma to be drawn across the dark polar cap by the prevailing steady state antisunward convection. Thus, a patch of plasma is isolated within the polar cap during the expansion episode and subsequently drifts antisunward. The depletion was found to be due primarily to the upward diffusion of ions into the topside. The increase in recombination rates due to frictional ion heating was found to be of only secondary importance in the formation of the depletion.

Millward et al. (1993b) used CTIP results to investigate the local thermospheric and ionospheric response to increased ion convection velocities in the auroral region. Joule heating causes a localised expansion of the neutral atmosphere. The upward air motion across the fixed pressure level surfaces requires a divergence in the horizontal wind field to preserve continuity. Changes in the horizontal neutral winds are also produced more directly by the action of increased Lorentz forcing. Thus an 'in-situ' disturbance is generated in the pressure level surfaces, the neutral temperature and vertical and horizontal wind fields. This disturbance was shown to propagate to mid-latitudes in the form of a large-scale travelling atmospheric disturbance (Millward et al., 1993a). More recently, Balthazor et al. (1997) investigated the relative importance of Joule heating and Lorentz forcing in generating TADs. The propagation of these TADs to equatorial latitudes was studied by Balthazor and Moffett (1997).

Ma and Schunk (1995) have used a stand-alone thermospheric model to investigate the effects of largescale ionospheric structure within the polar cap on the neutral atmospheric constituents. Their semi-empirical input ionospheric model consisted of a patch of ionisation, similar to that observed by Weber et al. (1984), artificially superimposed on a background steady state ionosphere and caused to drift antisunward at the plasma $\mathbf{E} \times \mathbf{B}$ convection velocity. They found that the increased ion drag force produced by the patch compared to the background ionosphere caused an increase in density due 
to the 'piling' up of neutral species at the leading edge of the 'patch'. This phenomenon was referred to as the 'snowplough' effect. Their modelling work, however, did not take into account fully the coupling between the neutral and charged species and was therefore not selfconsistent in this respect. Also no attempt was made to model the generation of the ionospheric patch.

We describe the thermospheric effects of the rapid polar cap expansion that produces the ionospheric structure already described by Idenden et al. (1997). The computations take into account both the effect of ionospheric structure on the neutral atmosphere and the disturbance in the neutral atmosphere produced by the expansion event itself. Because CTIP is fully coupled, the modulation of the ionosphere by the changing neutral composition, temperature and winds is also taken into account.

\section{Model description}

The modelling results we shall describe have been obtained using the coupled thermosphere/ionosphere/ plasmasphere model (CTIP) (Fuller-Rowell et al., 1996; Millward et al., 1996). The model was developed at Sheffield University and University College London in the UK and Space Environment Lab, Boulder, Colorado in the USA. A fully time-dependent high-latitude electric field model has since been incorporated (Idenden et al., 1996). Because these models have been described in detail elsewhere, it is our intention here to give only the essential features and inputs relevant to the present work. Since in this work we concern ourselves only with the high latitude regime, we do not describe in any detail the plasmaspheric code which calculates number densities and field aligned velocities for $\mathrm{O}^{+}$and $\mathrm{H}^{+}$within the $L=3$ shell. This code is described in detail by Millward et al. (1996).

The high latitude ionosphere code solves the coupled equations of continuity and momentum on straight 'open' field lines for the major species in the $\mathrm{F}$ layer and topside, $\mathrm{O}^{+}$and $\mathrm{H}^{+}$, down to latitudes of $\pm 35^{\circ}$. At each time step (1 min for the work described here) ion densities and field-aligned velocities are calculated by an implicit numerical scheme on a grid of field lines which extend from an altitude of 100 to $10000 \mathrm{~km}$ and whose positions are fixed in longitude and latitude with separations of $18^{\circ}$ and $2^{\circ}$ respectively. The computations include the effects of $\mathbf{E} \times \mathbf{B}$ convection, ion-ion collisions, ion neutral collisions, thermal diffusion, gravity, ion and electron partial pressure gradients, and chemical and photoproduction and loss mechanisms. Ion temperatures are calculated by solving the energy balance equation, which assumes that the rate at which heat is gained from the electron gas and by frictional heating is equal to the rate at which it is lost to the neutral air.

The thermospheric code solves the coupled momentum, continuity and energy equations by an explicit numerical scheme. Neutral temperatures, winds and densities are calculated on a global grid with the same horizontal resolution as the ionospheric code and with 15 fixed pressure levels. The first pressure level is fixed at
$80 \mathrm{~km}$ and $1.038 \mathrm{~Pa}$ and the remainder are separated by one scale height up to an altitude between approximately 400 and $600 \mathrm{~km}$ depending on conditions. The number densities of the major thermospheric species $\mathrm{O}, \mathrm{O}_{2}$, and $\mathrm{N}_{2}$ are calculated by solving the continuity equation for the mass mixing ratio and the general diffusion equation. The calculation of coupling terms such as the ion drag force (Lorentz force), and Joule heating require ionospheric densities and conductivities which are passed to the thermospheric code from the ionospheric code at high latitudes and from the plasmaspheric code inside the $L=3$ shell. Conversely, for the charged constituents, calculations of the heat exchange with the neutral gas, ion-neutral frictional heating, field aligned ion-neutral momentum transfer, and chemical production and loss rates require thermospheric temperatures, composition, densities, and winds. These parameters are passed to the high-latitude ionospheric and plasmaspheric codes from the thermospheric code.

The fully time-dependent electric field model has been described by Idenden et al. (1996). Electric field values are derived by solving Laplace's equation with boundary conditions specified on a circular polar cap boundary. Flux may enter and leave the polar cap through the throat on the dayside and the night side exit only. The required inputs to the model are the initial polar cap radius; the positions and widths of the throat and night exit regions; and the voltages across the two flux gaps, $\mathrm{V}_{D}$ and $\mathrm{V}_{N}$ respectively. If the dayside voltage exceeds the nightside voltage, the polar cap expands since the increasing flux within it is incompressible. Similarly, it contracts if $\mathrm{V}_{N}>\mathrm{V}_{D}$. The polar cap radius is calculated consistently with the integrated time histories of $\mathrm{V}_{N}$ and $\mathrm{V}_{D}$.

\section{Model starting conditions and inputs}

The model computations were for December solstice with moderate solar $(\mathrm{F} 10.7=165$.$) and magnetic \left(K_{p}=3-\right)$ activity. Winter solstice in the Northern Hemisphere was considered in the first place as being the season in which ionospheric structure in the polar cap is most frequently observed (Coley and Heelis, 1995). There is also some evidence to suggest that patches within the polar cap are remnants of the well-known tongue of ionisation (e.g. Bowline et al., 1996). Thus the polar cap expansion began at 1900 UT when the tongue of ionisation in model data was well formed (Idenden et al., 1997). This time also lies in the period identified by Buchau et al. (1985) during which patch occurrence is most frequent in December and coincides with the UT at which a patch was observed by Hill (1963) using a large array of ionosondes.

The initial starting conditions were obtained by running the model under steady state conditions for a number of days until equilibrium in the diurnal sense was obtained. The polar cap potential drop $\left(\mathrm{V}_{p c}\right)$ during the run was set to $46 \mathrm{kV}$. The convection pattern fitted to the empirical model of Foster et al. (1986) with precipitation index 6 which results in a polar cap radius $\left(\mathrm{R}_{p c}\right)$ of $14.9^{\circ}$. The throat and exit widths were set to $4 \mathrm{~h}$ and $6 \mathrm{~h}$ of local time, respectively. Input precipitation patterns were those 
derived empirically from TIROS satellite measurements by Fuller-Rowell and Evans (1987) and matched to the relevant Foster convection pattern.

At 1912 UT, the voltage across the throat was increased steadily over a $5 \mathrm{~min}$ interval from its steady state value of $46 \mathrm{kV}$ to a maximum value of $150 \mathrm{kV}$. Simultaneously, the nightside voltage was set to zero so that the polar cap expands rapidly. $\mathrm{V}_{D}$ was held at $150 \mathrm{kV}$ for a further $10 \mathrm{~min}$. Both dayside and nightside voltages were then set to $75 \mathrm{kV}$, the larger polar cap potential reflecting the increased polar cap radius of $16.8^{\circ}$. The convection patterns before, during, and after this event are shown in Fig. 1. Velocity vectors are shown in the Earth-fixed (corotating) frame of reference. Prior to the expansion, the classic, two-celled convection pattern prevails (Fig. 1a). At 1918 UT during expansion (Fig. 1b), large flows of about $2.3 \mathrm{~km} \mathrm{~s}^{-1}$ are seen through the throat region. Nightside flows are very much reduced and are primarily meridional as the plasma moves with the expanding boundary. Subsequent to the expansion (Fig. 1c), the steady state pattern prevails again with increased polar cap radius. During polar cap expansion, the input precipitation auroral oval is expanded in proportion to the polar cap radius change.

To discern more clearly the effects of the short lived energy impulse into the thermosphere, it is necessary to calculate the perturbations from a reference model run in which no rapid polar cap expansion has occurred. In this case, however, the derivation of such a model run is problematic: the electric field configuration after the expansion $\left(\mathrm{V}_{p c}=75 \mathrm{kV}, \mathrm{R}_{p c}=16.8^{\circ}\right)$ is different to the configuration before it $\left(\mathrm{V}_{p c}=46 \mathrm{kV}, \mathrm{R}_{p c}=14.9^{\circ}\right)$. After the expansion phase, perturbations from a steady state run with the same initial inputs $\left(\mathrm{V}_{p c}=46 \mathrm{kV}\right.$, $\mathrm{R}_{p c}=14.9^{\circ}$ ) as the expansion run will show both the transient effects of the expansion episode, when there are rapid flows through the throat, and the longer term effects due to these differences. It is also not appropriate to use as reference a diurnally reproducible simulation with $\mathrm{V}_{p c}=75 \mathrm{kV}$ and $\mathrm{R}_{p c}=16.8^{\circ}$. The difference between such a reference run and the initial starting conditions is very large and convergence of the expansion run and such a reference run would take days rather than hours. The large long-term differences would mask the more transient effects of the polar cap expansion phase. To obtain a more suitable reference, we began a simulation at 1800 UT (i.e. 1 hour prior to the expansion simulation) with the same diurnally reproducible initial conditions as the expansion run, but used the post-expansion steady state inputs, i.e. $\mathrm{V}_{p c}=75 \mathrm{kV}$ and $\mathrm{R}_{p c}=16.8^{\circ}$. The simulation was run for a further $4 \mathrm{~h}$. Perturbations post-expansion (after 1927 UT) were calculated with respect to this reference run.

\section{Results}

\subsection{Ionosphere}

In this section we summarise the results of Idenden et al. (1997) who describe the ionospheric structure resulting

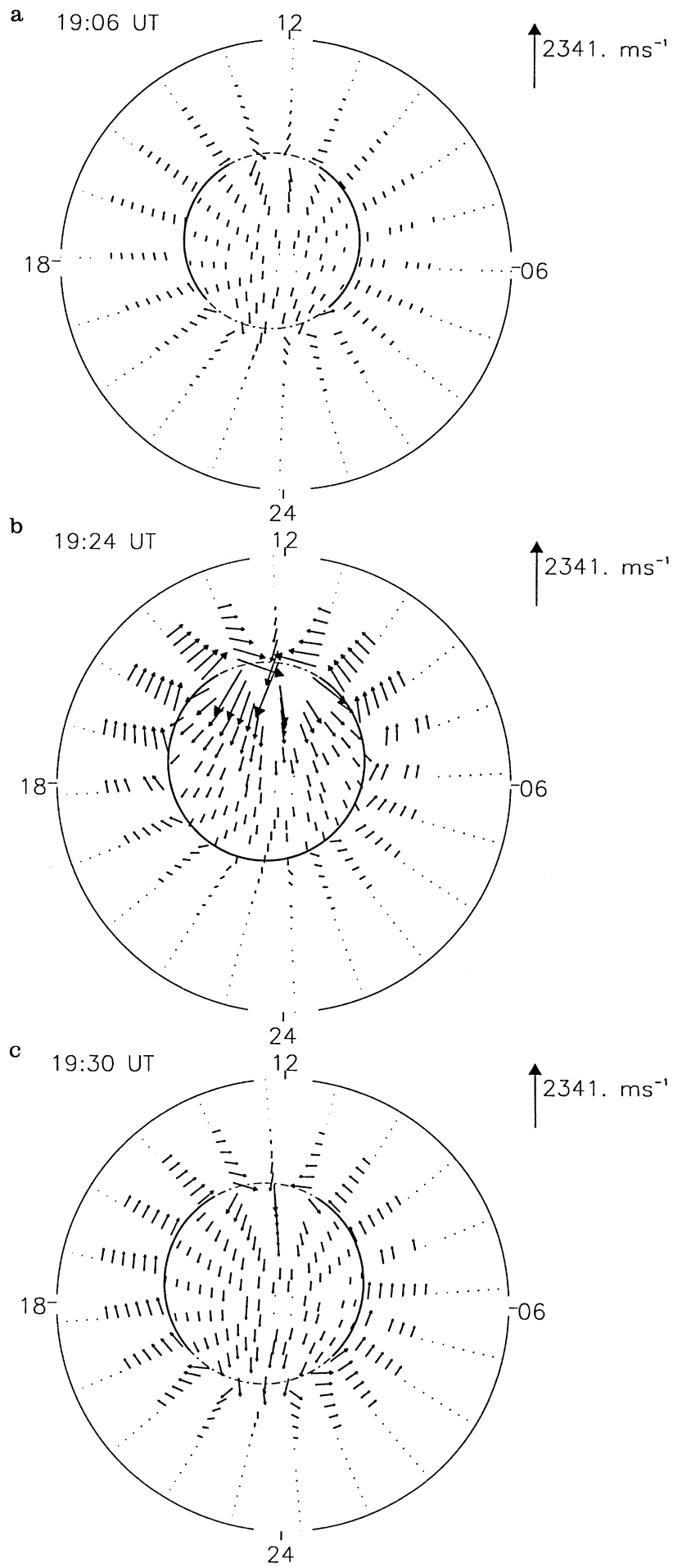

Fig. 1a-c. Input ion convection velocities at a 1906 UT, before the expansion event, b 1924 UT, during the event and c 1930 UT, after the event. The boundary of the plots is at $50^{\circ}$ latitude, and vectors on each have the same scale. The solid thick circle shows the polar cap boundary. The dot-dashed line indicates the flux gaps through which plasma may enter and leave the polar cap

from the rapid polar cap expansion. The reader should refer to the afore-mentioned reference for a more detailed discussion. We repeat the essential points here to put the thermospheric results in context, particularly 
in reference to the effects of the ionospheric structure on the thermosphere.

Figure 2 shows contour maps of NmF2 at 1906 UT prior to the polar cap expansion, and at 1930 UT and 2000 UT both after the expansion. At 1906 UT (Fig. 2a), the tongue of ionisation is well formed and stretches from the sunlit sub-auroral region well across the polar cap, the majority of which is in darkness. The positions of the terminators at ground and at $300 \mathrm{~km}$ altitude are marked by white dashed and dotted lines respectively. By the end of the polar cap expansion, at 1930 UT (Fig. 2b), a narrow depletion in $\mathrm{NmF} 2$ has formed across the width of the throat region. During the polar cap expansion, rapid convective plasma drifts (Fig. 1b) increase ionneutral frictional heating in the $\mathrm{F}$ region and give rise to large upward field aligned flows from the $\mathrm{F}$ region and into the topside. The $\mathrm{F}$ region becomes depleted. Enhanced recombination rates due to the increased effective ion temperature also play a part in the formation of the ionospheric depletion, though to a lesser extent than field aligned flows. Thus a large patch of ionisation (a remnant of the TOI) is isolated within the polar cap. A cross section through the patch at 1930 UT (Idenden et al., 1997) reveals the following characteristics:

1. A maximum $\mathrm{NmF} 2$ in the patch of $9.7 \times 10^{11} \mathrm{~m}^{-3}$, approximately a factor 2 above the background.

2. The height of the F peak in the depleted region is increased to an altitude of approximately $420 \mathrm{~km}$ compared to $330 \mathrm{~km}$ within the patch.

3. Characteristics (1) and (2) result in the maximum ion density at $330 \mathrm{~km}$ within the patch being 5 times that in the trailing trough.

4. The scale length $\left(L=\left|[1 / N(\partial N / \partial x)]^{-1}\right|\right)$ for the spatial electron density gradient is much greater on the leading edge of the patch $(L \approx 2900 \mathrm{~km})$ than on the trailing edge $(L \approx 990 \mathrm{~km})$.

Thirty minutes later (Fig. 2c), the patch is still evident and has drifted further across the polar cap. However, the patch has become smaller and less distinct as compared to the background. These characteristics can be explained by the return (downward) flow of plasma along flux tubes that were previously depleted during the polar cap expansion. Thus the $\mathrm{F}$ region ion densities on these plasma flux tubes actually increase as they $\mathbf{E} \times \mathbf{B}$ drift across the dark polar cap. This tends to 'fill in' the depleted region. In the meantime flux tubes in the patch lose ions which recombine in the darkness and thus have their densities reduced.

Fig. 2a-c. Polar plots of $\mathrm{NmF} 2$ down to a latitude of $50^{\circ}$ at a 1906 UT, b 1930 UT c 2000 UT. NmF2 values in units of $10^{10} \mathrm{~m}^{-3}$ are shown by the colour bar. NmF2 values are also indicated by the plotted contours. Bold contours are at intervals of $20 \times 10^{10} \mathrm{~m}^{-3}$. The tickmarks show the direction in which $\mathrm{NmF} 2$ decreases. The bold circle shows the polar cap boundary. The positions of the terminators at the ground and at $300 \mathrm{~km}$ are marked by white dashed and dotdashed lines respectively a
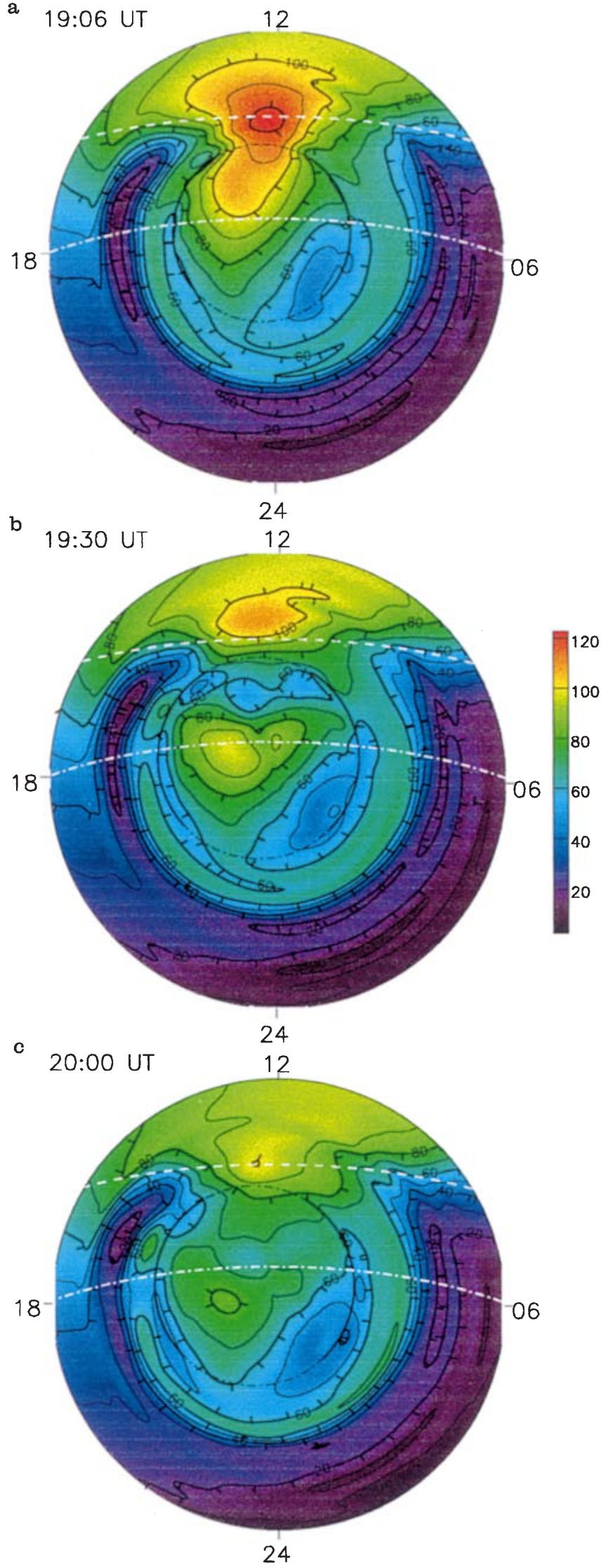


\subsection{Thermosphere}

During the rapid polar cap expansion, Joule heating of the neutral atmosphere due to rapid ion flows through the dayside convection throat result in an initially localised expansion of the atmosphere. The modelled temperature of the neutral gas is shown as a contour plot in a height versus UT plane in Fig. 3 a for the location $70^{\circ}$ latitude, $-100^{\circ}$ longitude. This is the position of the centre of the throat region midway through the expansion event. Also plotted are the heights of the fixed pressure levels. Clear disturbances in both the neutral temperature and the heights of the fixed pressure levels are evident and have their largest magnitudes at approximately 1924 UT. Altitude profiles of neutral temperature prior to the expansion event (1906 UT) and at the disturbance maximum (1930 UT) are plotted in Fig. 3b. The steady state neutral temperature has a fairly typical profile, increasing monotonically from $100 \mathrm{~km}$ to $300 \mathrm{~km}$ beyond which it flattens off rapidly, approaching a maximum temperature of approximately $1060 \mathrm{~K}$. The disturbance temperature profile, however, has a maximum of more than $1600 \mathrm{~K}$ at an altitude of about $420 \mathrm{~km}$. The position of this peak may be explained by the fact that the Joule heating rate per unit mass of neutral atmosphere maximises at or around the F2 peak, though the temperature peak will be relatively short lived as energy is transported from the heating region by advection, conduction and diffusion. It is clear from Fig. 3a, however, that the disturbance in pressure level heights increases monotonically with altitude.

The impulsive Joule heating event at the dayside throat gives rise to travelling atmospheric disturbances which propagate both equatorwards and polewards from the throat and which are much longer lived than the impulse itself. In this study we focus almost exclusively on the poleward travelling disturbance. This disturbance is most easily seen in perturbations from the reference run. The colour plots in Fig. $4 a$, b show the

a 19:06 19:25 19:45 20:04 20:23 20:43 21:02 21:21 21:41 22:00

UT (hrs)

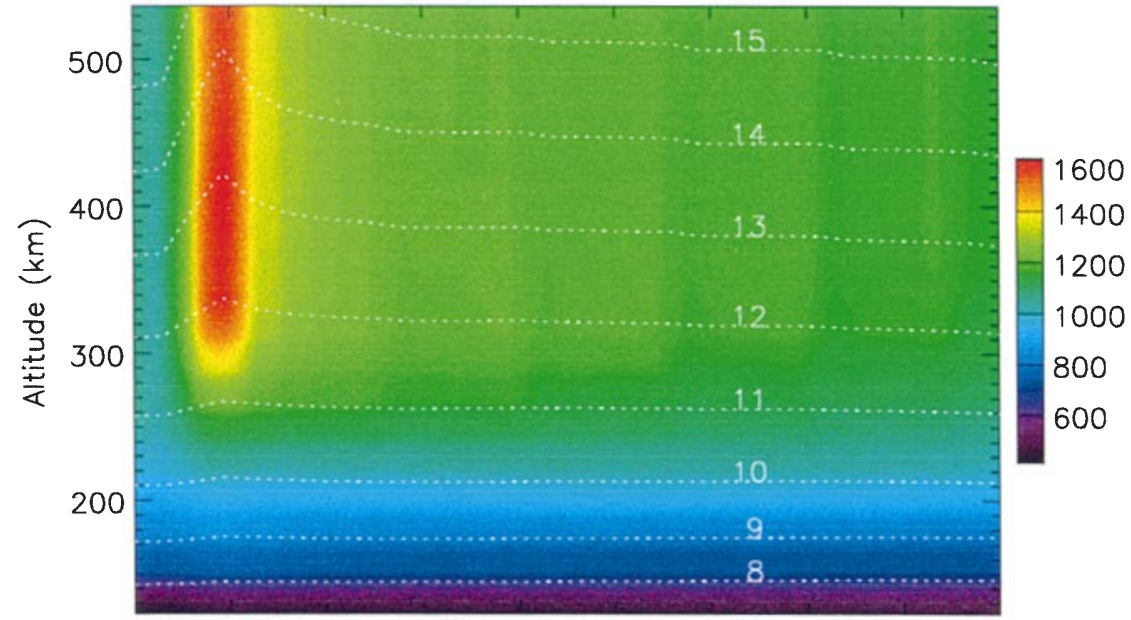

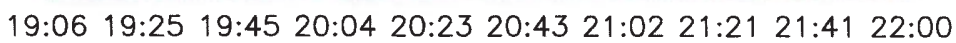

UT (hrs)

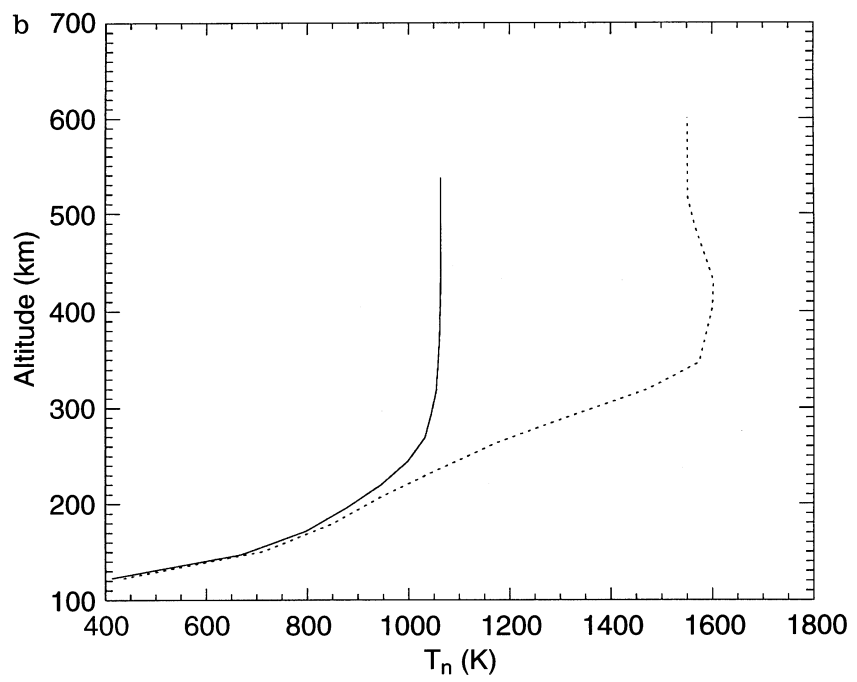

Fig. 3a. Time history plot of the altitude profile of the neutral temperature above the centre of the dayside throat $\left(70^{\circ}\right.$ latitude, $100^{\circ} \mathrm{W}$ longitude). The white dotted lines show the heights of the fixed pressure levels 8 to 15 . b Altitude profiles of neutral temperature at the centre of the dayside throat at 1906 UT (solid line) and 1924 UT (dotted line) 

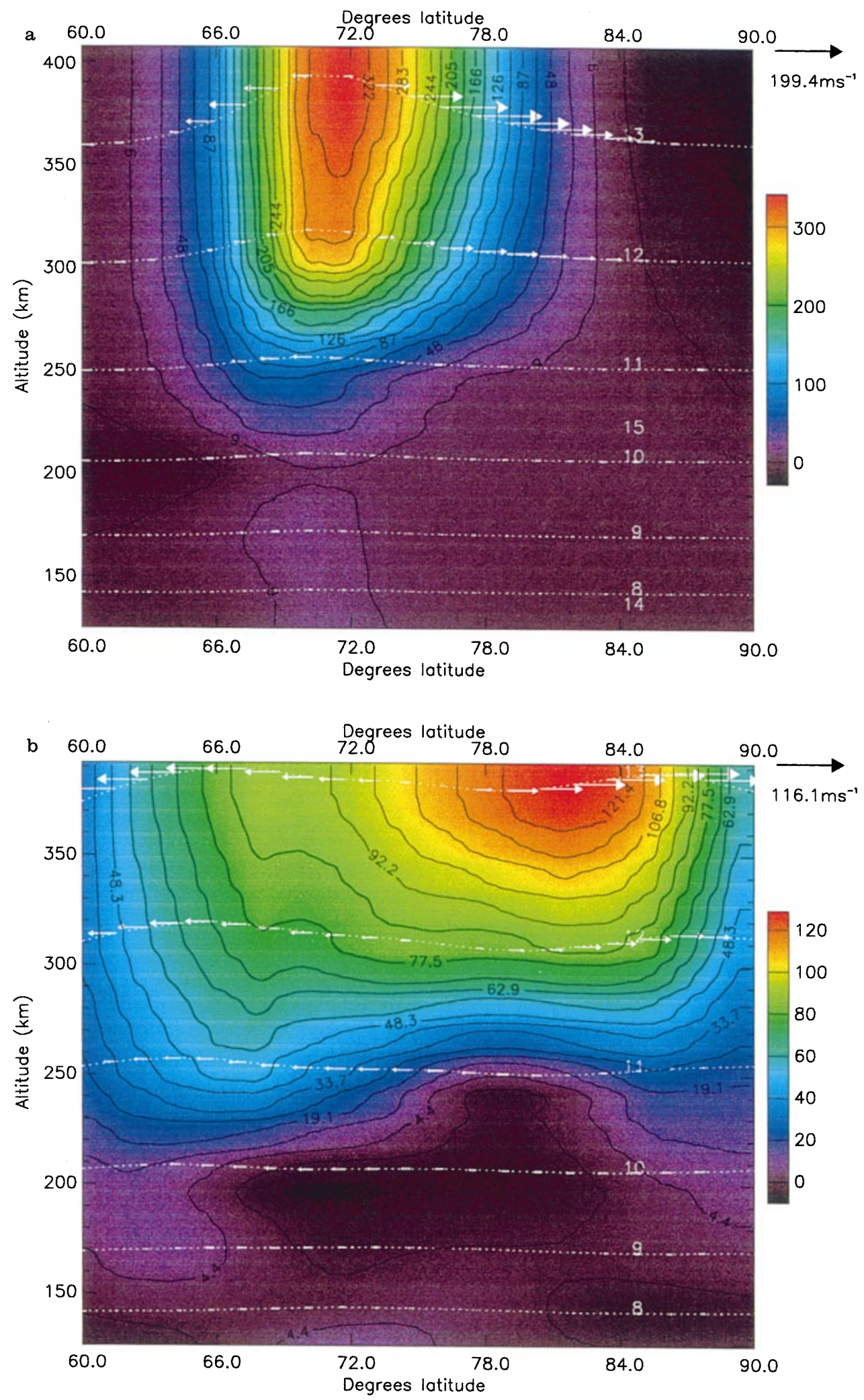

Fig. 4a, b. Altitude $/ 100^{\circ} \mathrm{W}$ meridian cross section plot of neutral temperature perturbations at a 1927 UT and b 1942 UT. The colour scales for each are different and are shown by the colour bar to the right of each (values in $\mathrm{K}$ ). Dotted lines show the perturbations in the heights of the fixed pressure levels multiplied by factors of $\mathbf{a} 1$ and $\mathbf{b} 3$ and each offset by their mean height. Plotted vectors show the perturbation in the meridional wind speed

neutral temperature perturbation in the meridian altitude plane through the centre of the flux gap $\left(-100^{\circ}\right.$ longitude) at 1927 UT and 1942 UT respectively. The 8 dotted lines show the perturbation in the heights of pressure levels 8 to 13 each offset by the height of the reference level averaged over the extent of the plot. The vectors show the change in the meridional component of the neutral wind velocity. At 1927 UT (Fig. 4a), just after the end of the expansion event, the disturbance in the neutral temperature is located close to the 
convection throat at $70^{\circ}$ latitude. The peak pressure level perturbation is approximately colocated with the temperature perturbation due to the localised thermal expansion of the atmosphere. Neutral winds are driven both equatorwards and polewards to either side of the pressure bulge. The poleward winds, however, are much larger in magnitude due to Lorentz forcing: Equatorward of the bulge, poleward Lorentz forcing produced by the enhanced ion velocities acts in the opposite sense to pressure gradient forces and reduces the horizontal velocity perturbation. Poleward of the bulge, Lorentz forcing and pressure gradient forces work together to produce greatly enhanced winds, with a maximum meridional perturbation of pressure level 13 (approximately $370 \mathrm{~km}$ ) of $200 \mathrm{~ms}^{-1}$. At 1942 UT (Fig. 4b), 17 min after the end of the expansion event, the temperature perturbation peak has travelled approximately $13^{\circ}$ poleward to $83^{\circ}$ latitude. The pressure level disturbance peak leads the temperature disturbance slightly, though, as we shall show, at later UTs they are in phase. The perturbation winds have their maximum value at the pressure bulge peak where the pressure gradient falls to zero and reverses direction.

A more global perspective on the passage of the atmospheric disturbance is provided by the snapshots in Fig. 5 which show polar plots of the perturbation in the
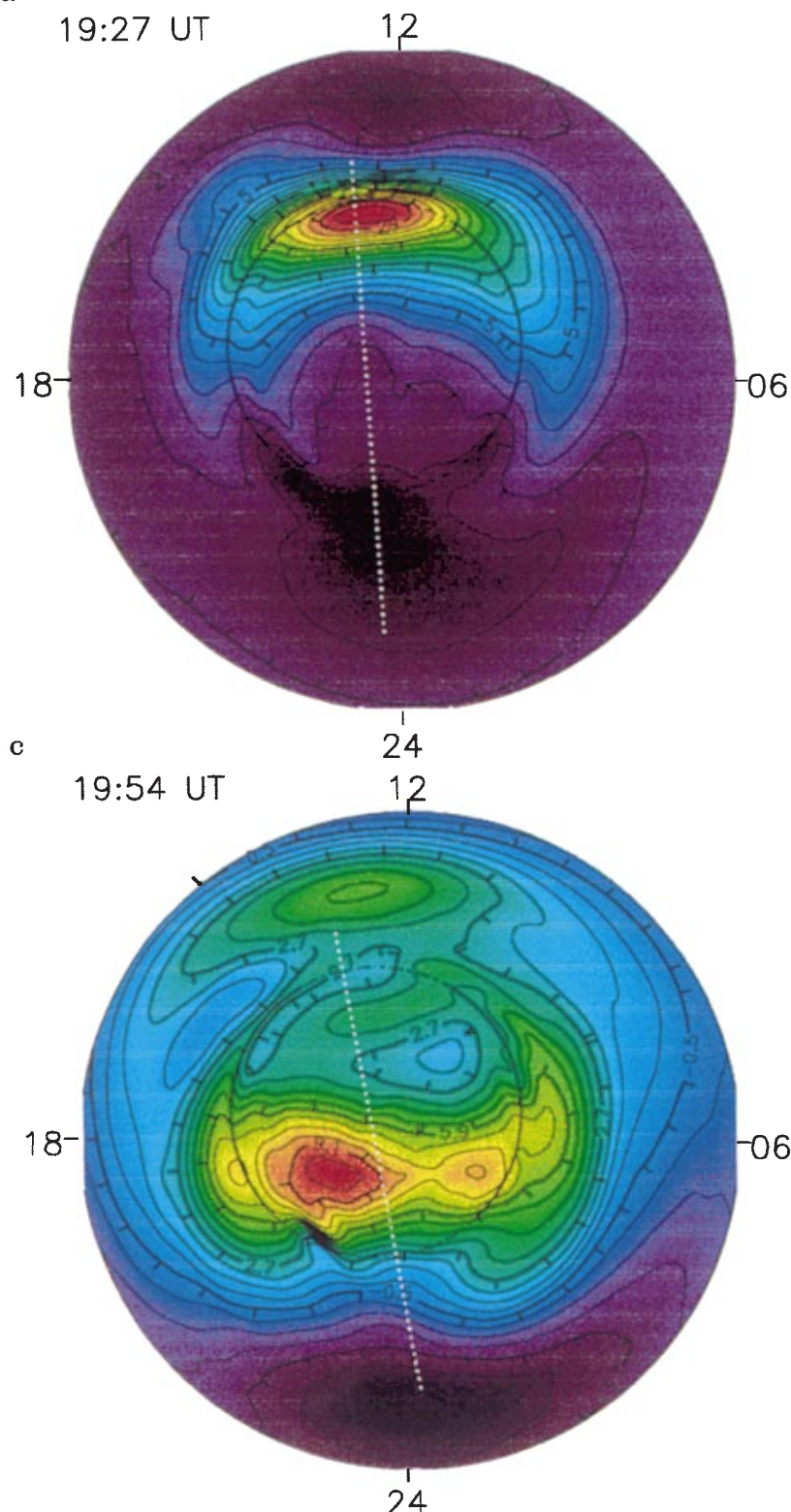

Fig. 5a-d. Polar plots of the perturbation in the height of pressure level 13 at a 1927 UT, b 1942 UT, c 1954 UT, and d 2006 UT. The boundary for all plots is at $50^{\circ}$ latitude. The colour scale is different for each plot and is shown in $\mathrm{km}$ by the colour bar to the right of each. The contours of perturbation heights are also plotted. The bold b

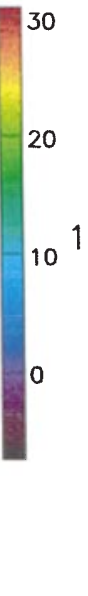

d
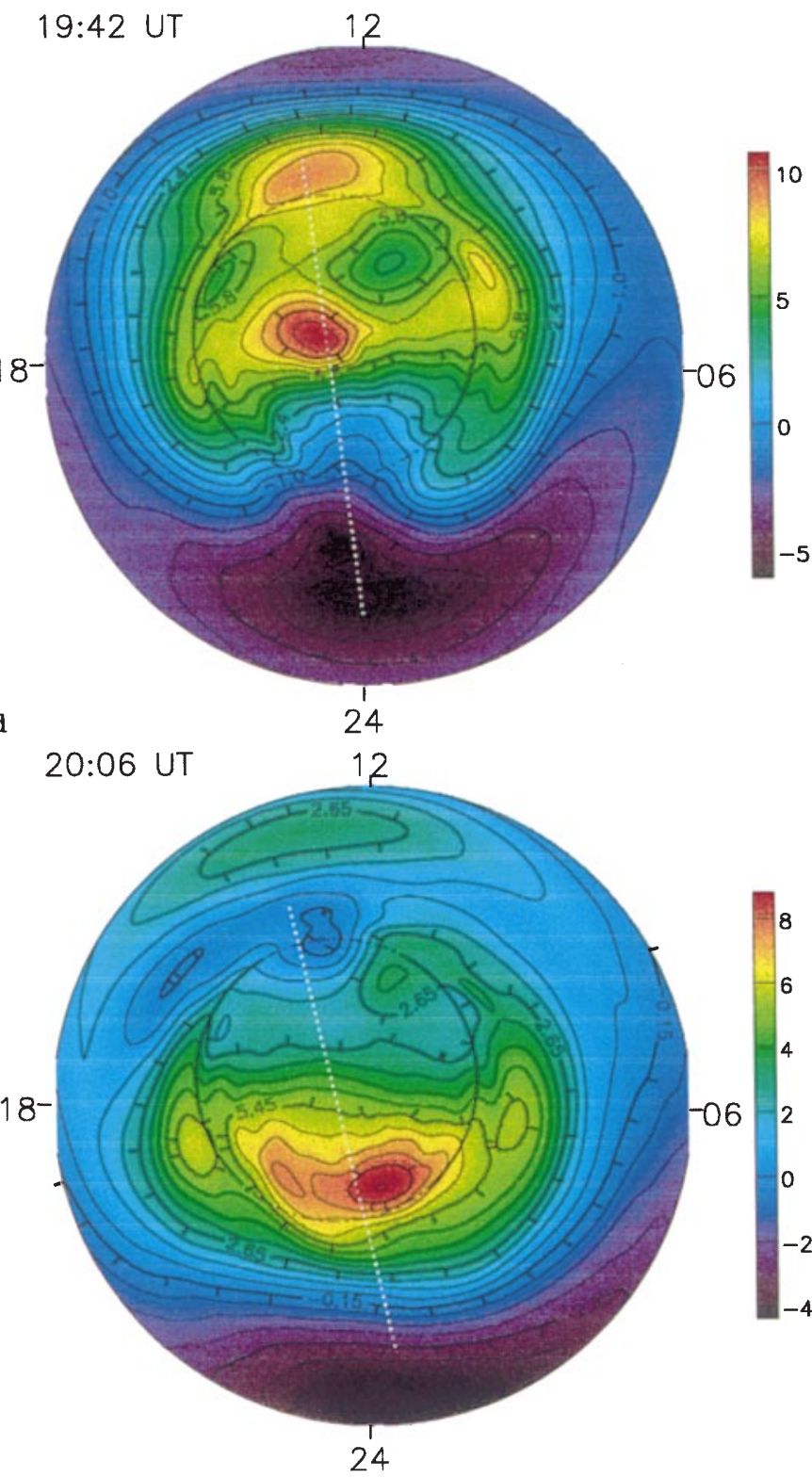

contours have their heights shown, and tick marks indicating the direction in which the perturbation height decreases. The dotted line shows the 'straight line' approximation to the path of the pressure disturbance that is used in Fig. 6 and 7 to obtain a velocity for the disturbance 
height of pressure level 13 (approximately $370 \mathrm{~km}$ altitude) at 4 different UTs. The disturbance clearly originates at the convection throat during polar cap expansion (Fig. 5a) and travels rapidly across the polar cap (Fig. 5b, c) exiting at 2006 UT (Fig. 5d). Caution should be used in comparing these plots, since a different colour scale has been used for each to allow the disturbance to be more easily distinguished. Thus it can be seen that the maximum height perturbation decreases from $31 \mathrm{~km}$ to $8.5 \mathrm{~km}$ over the 39 min spanned by these plots indicating a decay time of approximately $30 \mathrm{~min}$ for the pressure level disturbance amplitude. The approximate path of the disturbance across the polar cap and beyond is marked by the dotted line in Fig. 5 .

To estimate the velocity of the disturbance, we obtained values for a number of parameters along the path marked in Fig. 5 at each model output UT (every 3 min) after 1927 UT. Colour contour plots of perturbation values were then obtained in UT - path distance space. The neutral temperature perturbation at pressure level 13 is plotted in this form in Fig. 6. The temperature disturbance is evident as the ridge of enhanced temperature values that runs from bottom left to top right of the plot. The time lines that would be taken by disturbances travelling with and at a constant velocity of $750 \mathrm{~ms}^{-1}$ relative to the neutral gas and originating at the convection throat at 1924 UT are shown by the solid and dotted lines respectively. The temperature disturbance ridge follows closely the $750 \mathrm{~ms}^{-1}$ time line suggesting this to be a good estimate of the velocity of the temperature disturbance. Similar velocities are obtained from contour plots of the same format for the altitude and path-parallel component of horizontal velocity perturbations for gas on pressure level 13 (not shown). The features in these plots are summarised in Fig. 7 which shows the UT of maximum perturbation in each parameter at each point on the path. It can be seen that the disturbances in all of these parameters are in phase and follow approximately the same time line.

To make direct comparisons between our results and those of Ma and Schunk (1995) we interpolated our data from the fixed pressure level grid onto a fixed height of $300 \mathrm{~km}$ and calculated the thermospheric densities at this altitude. The perturbation densities are shown as a series of polar plots obtained at a number of UTs in Fig. 8. Also shown on each of these is the position of the plasma patch (marked by a diamond symbol) which was formed during the polar cap expansion and the position of the maximum perturbation in the height of pressure

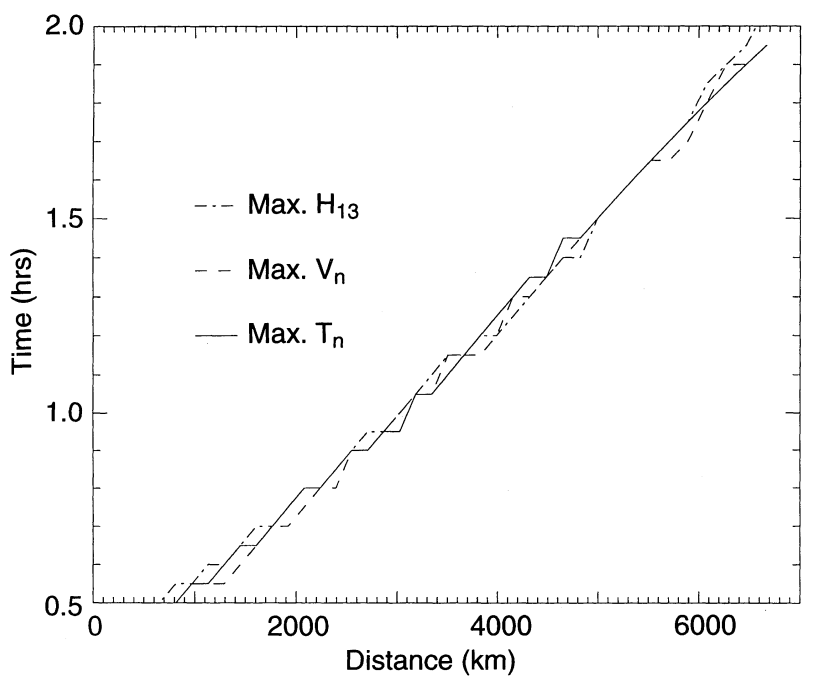

Fig. 7. Time lines for the motion of the disturbances in the height (dot-dashed line), temperature (solid line) and horizontal velocity (dashed line) for the neutral gas on pressure level 13

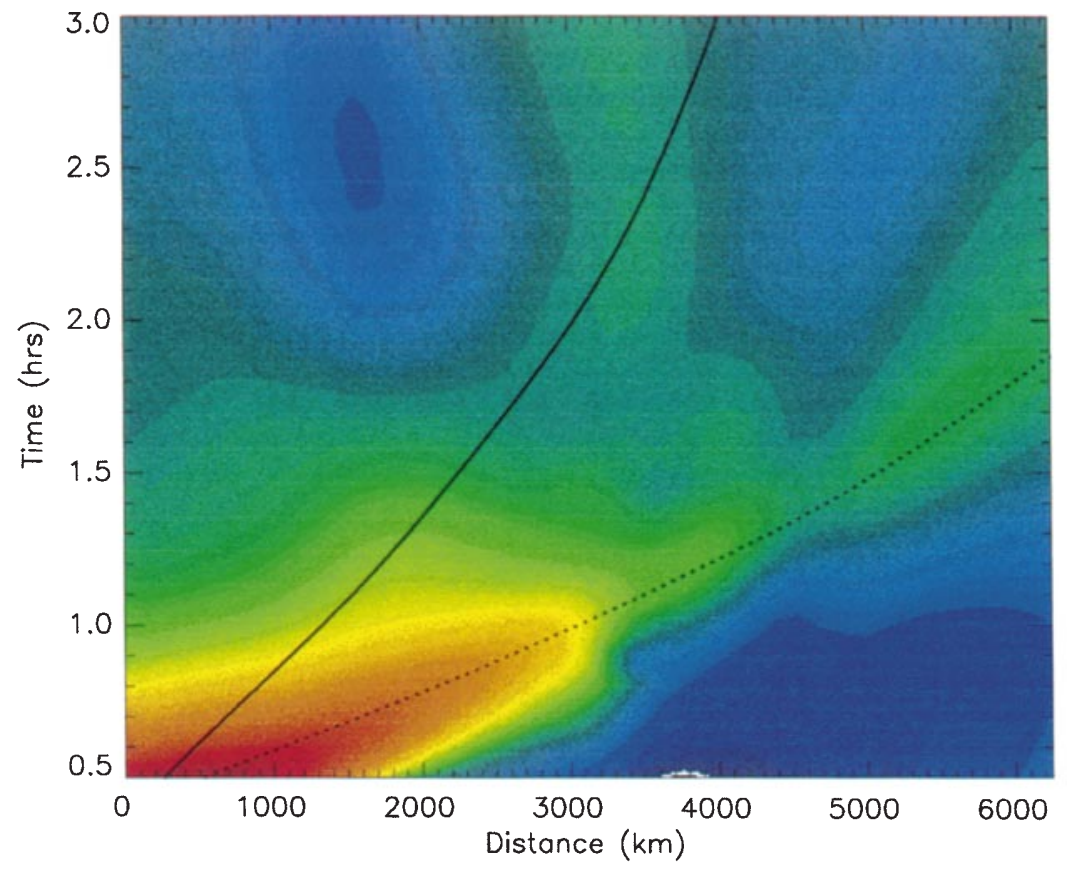

20

15

10

5

0
Fig. 6. Colour contour plot of the time history of the temperature perturbation on pressure level 13 along the approximate path of the pressure level disturbance. The horizontal axis indicates the distance from the throat along the path indicated in Fig. 5. The vertical axis indicates the time in hours after 1900 UT. The solid and dotted lines indicate the motion of a disturbance travelling at the neutral wind speed and at a velocity of $750 \mathrm{~ms}^{-1}$ relative to the neutral gas respectively 
a

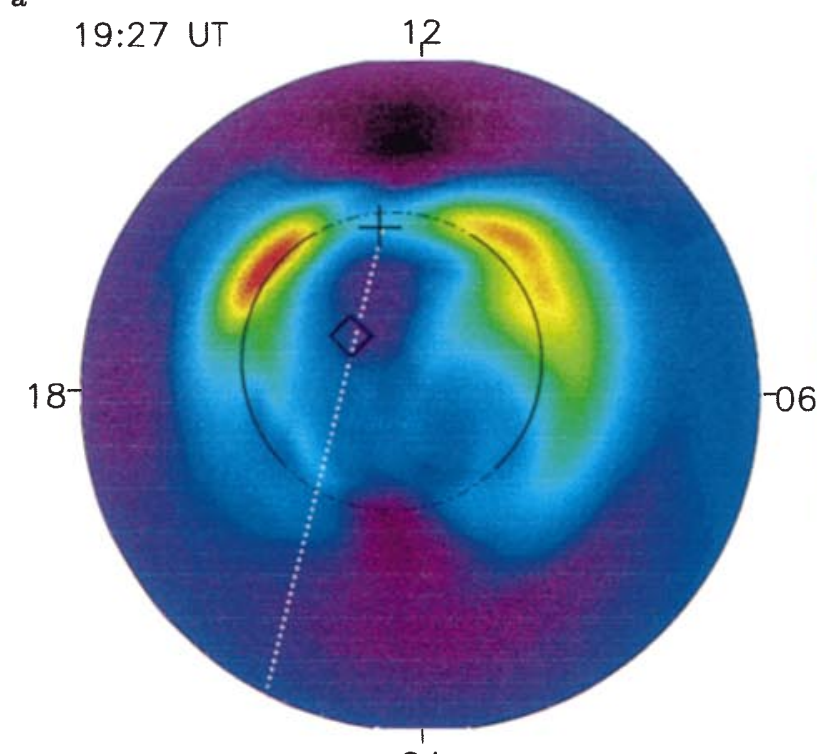

c

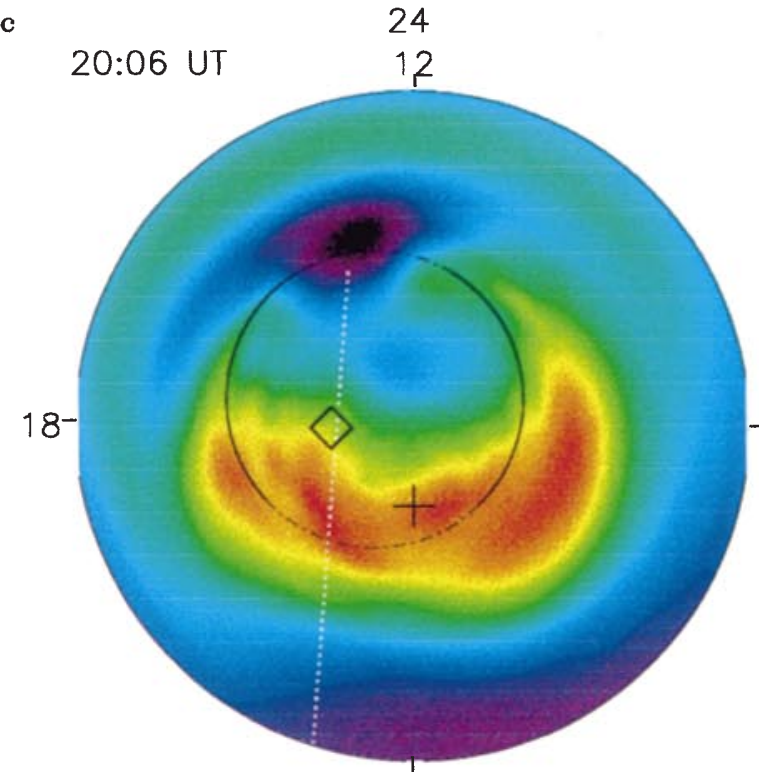

e

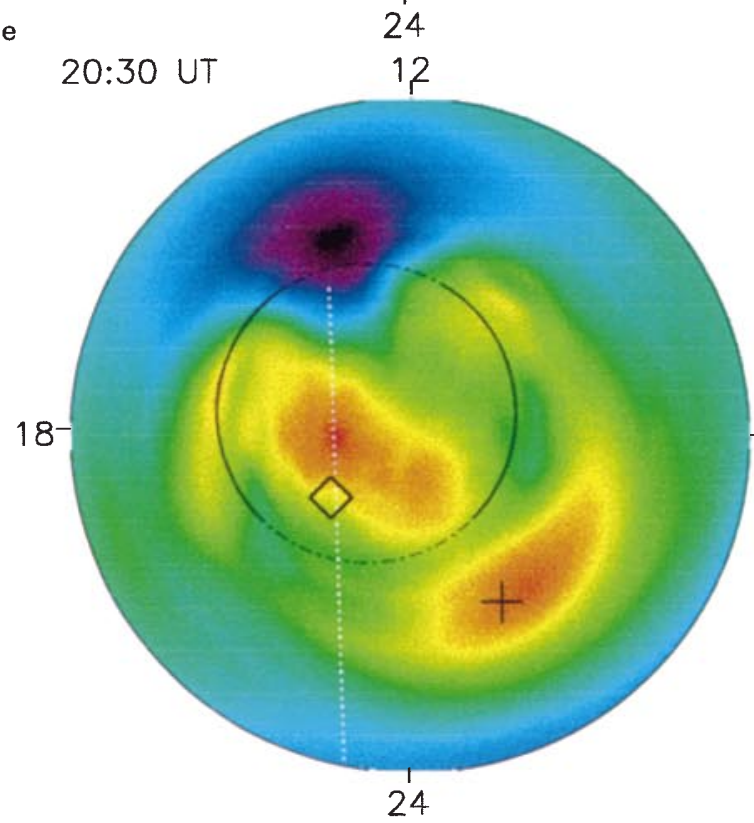

b
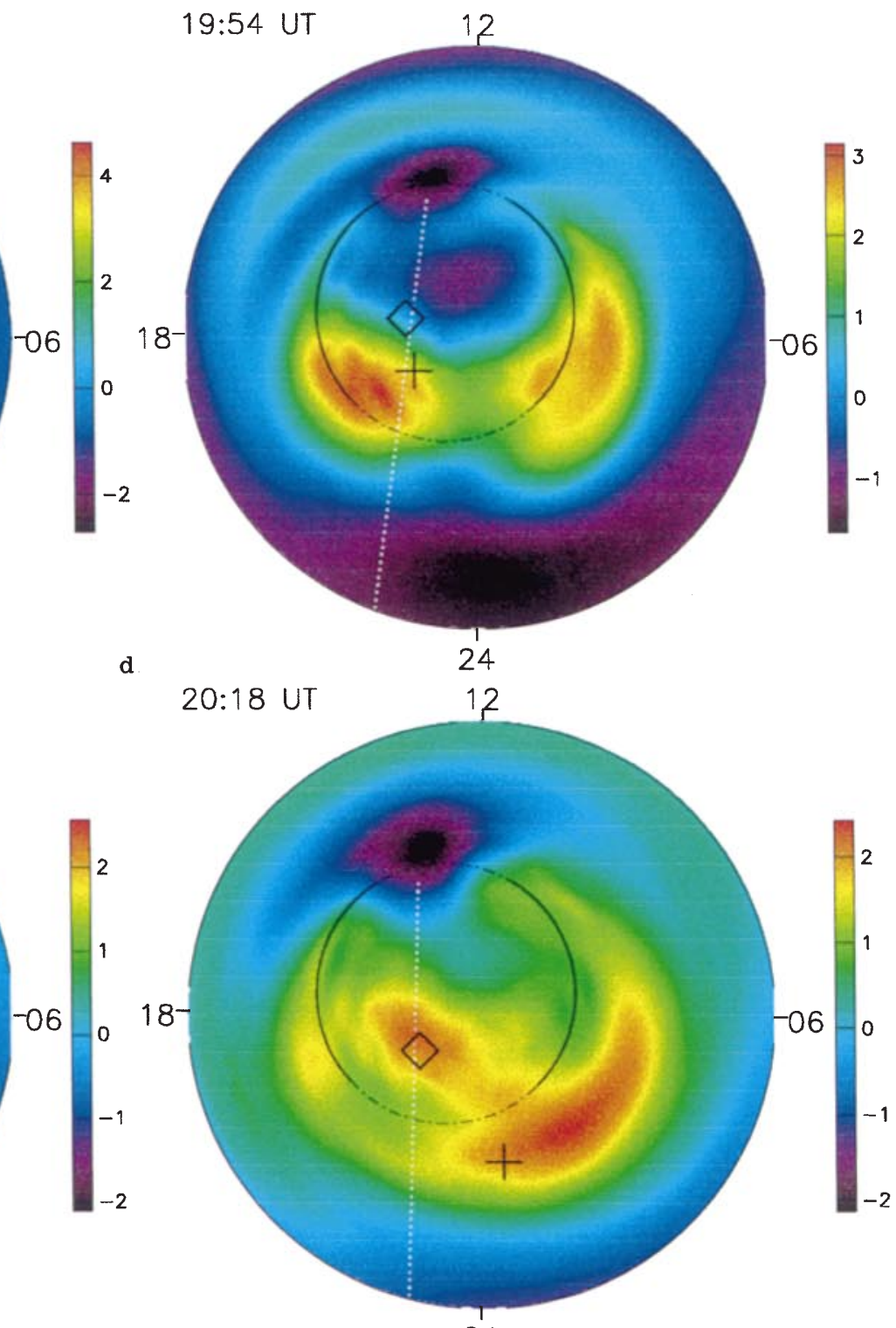

f

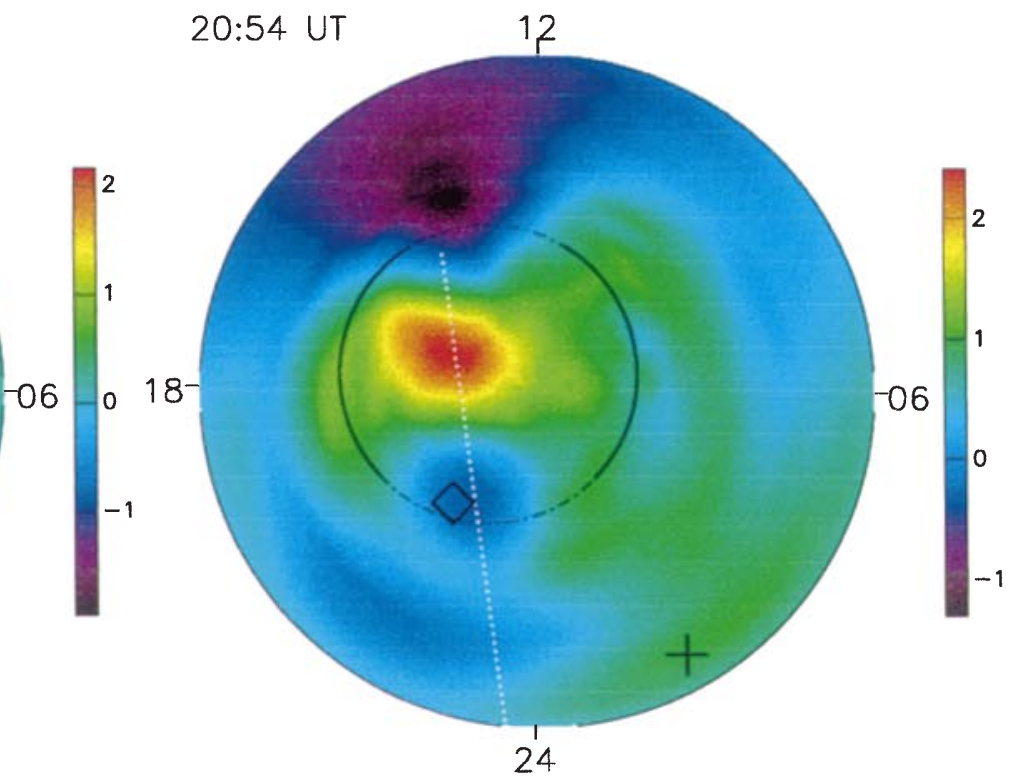


Fig. 8a-f. Polar plots of the perturbation in the neutral density at 300 $\mathrm{km}$ altitude at UTs of a 1927, b 1954, c 2006, d 2018, e 2030, and f 2054. The boundary of each plot is at a latitude of $50^{\circ}$. The colour scales vary and are shown by the colour bars to the right of each plot. Density perturbations are in units of $10^{-12} \mathrm{~kg} \mathrm{~m}^{-3}$. The cross and diamond symbol mark the position of the maximum pressure perturbation and maximum $\mathrm{NmF} 2$ in the plasma patch respectively. The dotted line marks the straight line approximation for the path taken by the plasma patch

level 13 (marked by a cross). The neutral density response at $300 \mathrm{~km}$ is evidently complex. A positive density perturbation originates at the throat at around 1927 UT (Fig. 8a) and is evident at subsequent UTs travelling anti-sunward across the polar cap and beyond (Fig. 8b-f). This disturbance appears related to the pressure bulge. The positions of each very nearly coincide at all UTs. The maximum density enhancement in this disturbance is $10 \%$. This feature is unlikely to be related to the plasma patch which travels much more slowly $\left(\mathrm{V} \approx 260 \mathrm{~ms}^{-1}\right)$

At 2018 UT (Fig. 8d), a distinct second disturbance in the neutral density is also evident and coincides at this UT with the patch density maximum. The two, however, do not appear related as this second disturbance appears to travel sunward, and at later UTs (Fig. 8e, f) no longer coincides with the patch. It seems likely that this second feature is due to the slow thermospheric response to the new set of steady input conditions rather than a transient response to the rapid polar cap expansion episode.

To demonstrate the lack of correspondence between either of these features and the plasma patch, we obtained values of the neutral density perturbation along the approximate straight line path of the plasma patch marked by the dotted line in Fig. 8 at each output UT. These values were then plotted in Fig. 9 in the timepath distance space using the same format as Fig. 6 . Also marked on this plot are the motions of the maximum $\mathrm{NmF} 2$ in the plasma patch (solid line) and the minimum $\mathrm{NmF} 2$ in the trailing trough (dotted line). No feature is evident in the neutral density whose motion follows that of the patch or the trough.

\section{Discussion and Summary}

It is evident from the results we report here that the polar cap thermospheric perturbations produced by the rapid polar cap expansion are dominated by the passage of a large scale travelling atmospheric disturbance. This disturbance is generated close to the convection throat by Joule heating and Lorentz forcing produced by the elevated ion velocities which are a feature of our expanding polar cap electric field model. The disturbance propagates across the polar cap at a speed of approximately $750 \mathrm{~ms}^{-1}$, and manifests itself as a positive pressure perturbation. Coincident with the pressure bulge is a positive temperature perturbation and horizontal neutral wind perturbation in the direction of propagation.

The rapid polar cap expansion also gives rise to ionospheric effects that have already been reported in a previous publication (Idenden et al., 1997). Most significant of these effects is the formation of a large-scale patch of ionisation which is similar in many aspects to that observed by Weber et al. (1984). The patch has a steep density gradient on its trailing edge, while the densities on the leading edge decline more slowly; hmF2 is greatly elevated on the trailing edge of the patch. Our patch, however, has densities at the F2 peak only twice

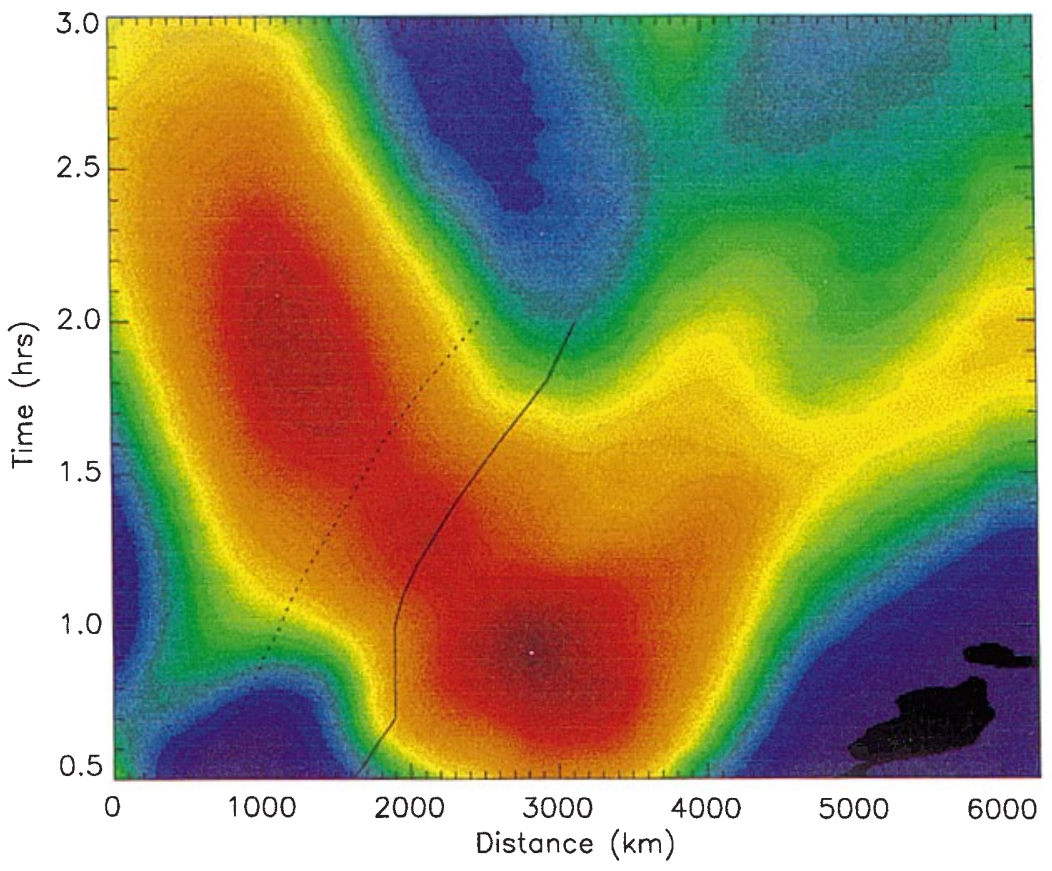

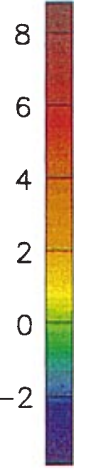

Fig. 9. Colour contour plot of the time history of the neutral density perturbation at $300 \mathrm{~km}$ along the approximate path of the plasma patch. The horizontal axis indicates the distance from the throat along the path indicated in Figure 8. The vertical axis indicates the time in hours after 1900 UT. The solid and dotted lines indicate the motion of the plasma patch and trailing trough respectively 
that of the background compared to a corresponding factor of approximately 8 for the Weber et al. (1984) patch. However, at a fixed height of $330 \mathrm{~km}$, the ion density at the centre of the patch is 5 times the density at the trailing edge.

We have examined the model neutral densities at a fixed height of $300 \mathrm{~km}$ in an attempt to discern the effects on the neutral atmosphere of the patch of ionisation. We do not, however, see evidence for the 'snowplough' effect suggested by the modelling results of Ma and Schunk (1995). The snowplough effect results in increased neutral densities on the leading edge of the patch due to the increased ion drag forces within the patch compared to those in front of its leading edge. There are a number of possible explanations for the differences between the results of the current modelling work and those of Ma and Schunk (1995):

1. In the modelling work we describe here thermospheric and ionospheric parameters are calculated self-consistently. Ma and Schunk's (1995) thermospheric model, however, is essentially a stand-alone model, so that changes in the thermospheric parameters in no way affect the artificially imposed ionosphere. This latter approach obviously ignores some important physical processes which are particularly important in dynamic events: as we have shown, Joule heating and ion drag forces on the neutral atmosphere give rise to neutral winds. These winds in turn can blow ions up or down the field line and thus modulate the ionosphere and in turn the Joule heating itself. Raising or lowering the ionosphere will change the neutral atmospheric chemical composition at the F2 peak, as indeed will the expansion and contraction of the neutral atmosphere itself and the resulting horizontal neutral winds which modulate composition by advection. In turn, it has been shown that the ion density at the $\mathrm{F} 2$ peak $(\mathrm{NmF} 2)$ is critically dependent on the ratio $[\mathrm{O}] /\left[\mathrm{N}_{2}\right]$ at the peak. Thus, the ionosphere/thermosphere is a highly coupled system and must be treated as such if realistic simulations are to be performed.

2. The modelling work of Ma and Schunk (1995) does not take into account the effects on the thermosphere of the initial disturbance responsible for generating the patch. Although we do not claim that our mechanism is the only one that operates, it seems likely that any realistic scheme for generating a patch must also create a significant thermospheric disturbance. In our modelling work, it is this disturbance that dominates the thermospheric perturbation, rather than the resulting ionospheric patch. The perturbation in neutral density at $300 \mathrm{~km}$ due to the travelling disturbance is approximately $10 \%$, similar to the maximum value $(12 \%)$ attributed to the snowplough effect by Ma and Schunk (1995).

3. The patch generated by the polar cap expansion has a lower density compared to the background than that imposed by Ma and Schunk (1995). At the F2 peak our patch has a maximum electron density twice that of the background, while that of Ma and Schunk is 5 times the background. Thus, one might expect the snowplough effect to be less evident in our results. At the trailing edge of our patch, however, hmF2 is greatly raised. At $330 \mathrm{~km}$, the electron density is a factor of 5 below the peak density at that altitude in the patch, in better agreement with Ma and Schunk's (1995) patch.

4. Our patch has a much steeper density gradient on its trailing edge than on its leading edge, the reverse of Ma and Schunk's (1995) patch. Our patch is in better agreement with that reported by Weber et al. (1984), who show results from an ionosonde above which $\mathrm{NmF} 2$ increases slowly as the patch approaches, and then declines rapidly after it has passed overhead. Thus on the leading edge of the patch the snowplough mechanism, which relies on the existence of a large ion drag gradient, is likely to be less effective.

5. Our patch lasts only approximately $2 \mathrm{~h}$. NmF2 at the trailing edge of the patch increases slowly as the patch convects across the polar cap due to the return flux of $\mathrm{O}^{+}$ions from the topside. The patch therefore becomes less distinct compared to the background. The patch imposed by Ma and Schunk (1995) however, lasts for the 3-4 h duration of their simulation during which time its density relative to background remains unchanged.

Acknowledgement. This work has been supported by PPARC under grant GR/K 06112.

Topical Editor D. Alcaydé thanks a referee for his help in evaluating their paper.

\section{References}

Balthazor, R. L., and R. J. Moffett, A study of atmospheric gravity waves and travelling ionospheric disturbances at equatorial latitudes, Ann. Geophysicae, 15, 1048, 1997.

Balthazor, R. L., R. J. Moffett, and G. H. Millward, A study of the Joule and Lorentz inputs in the production of atmospheric gravity waves in the upper thermosphere, Ann. Geophysicae, 15, 779, 1997.

Bowline, M. D., J. J. Sojka, and R. W. Schunk, Relationship of theoretical patch climatology to polar cap patch observations, Radio Sci., 31, 635, 1996.

Buchau, J., E. J. Weber, D. N. Anderson, H. C. Carlson Jr., J. G. Moore, B. W. Reinisch, and R. C. Livingston, Ionospheric structures in the polar cap: their origin and relation to $250 \mathrm{MHz}$ scintillation, Radio Sci., 20, 325, 1985.

Coley, W. R., and R. A. Heelis, Adaptive identification and characterisation of polar ionisation patches, J. Geophys. Res., 100, 23 819, 1995.

Foster, J. C., J. M. Holt, R. G. Musgrove, and D. S. Evans, Ionospheric convection associated with discrete levels of particle precipitation, Geophys. Res. Lett., 13, 656, 1986.

Fuller-Rowell, T. J., and D. S. Evans, Height integrated Pederson and Hall conductivity patterns inferred from the TIROSNOAA satellite data, J. Geophys. Res., 92, 7606, 1987.

Fuller-Rowell, T. J., D. Rees, S. Quegan, R. J. Moffett, M. V. Codrescu, and G. H. Millward, A coupled thermosphereionosphere model (CTIM), in STEP Handbook on Ionospheric Models, Ed. R. W. Schunk, pp. 217-238, Utah State University, Logan, Utah, 1996.

Hill, G. E., Sudden enhancements of F-layer ionization in polar regions, J. Atmos. Sci., 20, 492, 1963. 
Idenden, D. W., R. J. Moffett, S. Quegan, and T. J. Fuller-Rowell, Time dependent convection at high latitudes, Ann. Geophysicae, 14, 1159, 1996.

Idenden, D. W., R. J. Moffett, and S. Quegan, Ionospheric structure produced during a rapid polar cap expansion, J. Geophys. Res., 102, 14 581, 1997.

Ma, Ti-Ze, and R. W. Schunk, Effect of polar cap patches on the polar thermosphere, J. Geophys. Res., 100, 19 701, 1995.

Millward, G. H., R. J. Moffett, S. Quegan, and T. J. Fuller-Rowell, Effects of an atmospheric gravity wave on the midlatitude ionospheric F layer, J. Geophys. Res., 98, 19 173, 1993a.

Millward, G. H., S. Quegan, R. J. Moffett, and T. J. Fuller-Rowell, A modelling study of the coupled ionospheric and thermos- pheric response to an enhanced high-latitude electric field event, Planet. Space Sci., 41, 45, 1993 b.

Millward, G. H., R. J. Moffett, S. Quegan, and T. J. Fuller-Rowell, A coupled thermosphere-ionosphere-plasmasphere model, CTIP, in STEP Handbook on Ionospheric Models, Ed. R. W. Schunk, pp. 239-280, Utah State University, Logan, Utah, 1996.

Weber, E. J., J. Buchau, J. G. Moore, J. R. Sharber, R. C. Livingstone, J. D. Winningham, and B. W. Reinisch, F-layer ionisation patches in the polar cap, J. Geophys. Res., 89, 1683, 1984. 